\title{
VIOLÊNCIA DOMÉSTICA CONTRA MULHERES EA RELAÇÃO POSSÍVEL COM INDICADORES ECONÔMICOS E SOCIAIS
}

\author{
JANAEL DA SILVA ALVES \\ Graduado em Geografia, mestre em Gestão Pública e Sociedade, especialista nas áreas de Gestão em Segurança Pública, \\ Planejamento e Gestão de Trânsito, Direito Administrativo e Educação em Direitos Humanos. Sargento na Policial Militar \\ de Minas Gerais. \\ País: Brasil Estado: Minas Gerais Cidade: Varginha \\ Email de contato: janael10@yahoo.com.br ORCID: https://orcid.org/0000-0002-1766-8600
}

\section{RESUMO}

Os crimes de violência doméstica contra a mulher são caracterizados como aqueles que acontecem no âmbito das relações familiares. Esta modalidade criminosa veio a ser considerada uma política pública a partir do ano de 2006, com a edição da lei nominada Maria da Penha, quando os crimes de violência doméstica passaram a ser monitorados com maior acuidade pelos governos e órgãos de segurança pública. Este trabalho realiza uma análise descritivo comparativa dos registros de crimes de violência doméstica contra mulheres ocorridos no ano de 2018 nas regiões Sul e Norte do Estado de Minas Gerais, buscando a possível relação entre os indicadores socioeconômicos dessas regiões e os registros de violência doméstica. O trabalho foi desenvolvido utilizando-se fontes de dados oficiais divulgados por meio de sites do governo de Minas Gerais. Descobriu-se a existência de uma correlação entre os indicadores econômicos sociais favoráveis e o alto número de registros de ocorrências de violência doméstica.

Palavras-chave: Violência doméstica. Violência intrafamiliar. Segurança pública. Sul de Minas Gerais.

\section{ABSTRACT \\ DOMESTIC VIOLENCE AGAINST WOMEN AND THE POSSIBLE RELATIONSHIP WITH ECONOMIC AND SOCIAL INDICATORS}

The crimes of domestic violence against women are characterized as those that occur in the context of family relations, this criminal mode came to be considered a public policy from the year 2006, with the edition of the law named Maria da Penha, when crimes of domestic violence began to be monitored more closely by governments and public security agencies. This work a comparative descriptive analysis of the records of crimes against domestic violence against women occurred in 2018 in the South and North of the State of Minas Gerais will be carried out, seeking a possible relation between the socioeconomic indicators of these regions and the records of domestic violence. The work was developed using official data sources published through Minas Gerais government websites. It was found that there is a correlation between favorable social economic indicators and the high number of records of occurrences of domestic violence.

Keywords: Domestic violence. Intrafamily violence. Public security. South of Minas Gerais. 


\section{INTRODUÇÃO}

O presente artigo apresenta os números de registros de violência doméstica contra mulheres nas regiões Sul e Norte do Estado de Minas Gerais e faz uma análise comparativa entre essas duas regiões. A região Sul apresenta indicadores socioeconômicos dentre os mais positivos do Estado, ao contrário, a região Norte apresenta indicadores dentre os mais desfavoráveis. Foi realizada uma análise comparativa entre as regiões mencionadas buscando uma possível correlação entre a modalidade criminosa da violência doméstica contra a mulher e os indicadores socioeconômicos dessas regiões do Estado de Minas Gerais, buscando também verificar se pelos registros oficiais que são divulgados por meio de órgãos de segurança pública é possível apontar a existência da correlação entre as condições socioeconômicas dos indivíduos e os crimes de violência doméstica.

A Lei Maria da Penha considera crime de violência doméstica e familiar contra a mulher "qualquer ação ou omissão baseada no gênero que the cause morte, lesão, sofrimento físico, sexual ou psicológico e dano moral ou patrimonial" (BRASIL, 2011, p. 16).

Ressalta-se a existência de uma alta taxa de subnotificação de crimes que, conforme MacDonald (2002), é uma variável importante a ser considerada na análise criminal, e ainda Madalozzo (2011), que destaca em seu trabalho a pesquisa do Instituto Futuro Brasil, que revela, por exemplo, no caso da cidade de São Paulo, um índice de subnotificação de 68\% dos crimes. Esse é um fator de limitação nos estudos de análise criminal, sendo assim, o presente trabalho irá utilizar os dados e as estatísticas disponíveis pelos canais oficiais do governo, considerando para o presente estudo os crimes de violência doméstica contra a mulher que foram notificados e registrados pelos órgãos de segurança pública do Estado de Minas Gerais.

\section{METODOLOGIA}

O trabalho trata-se de uma pesquisa exploratória de abordagem quantitativa a partir de fontes secundárias e descreve o fenômeno da violência doméstica em duas regiões de planejamento do Estado de Minas Gerais. O Estado, para fins de administração e planeamento nas mais diversas áreas, é dividido em 17 regiões, conforme Minas Gerais (2019); já para fins de coordenação e controle, especificamente na área da segurança pública, o Estado é dividido em 19 Regiões Integradas de Segurança Pública (RISPs).

O trabalho se propõe fazer uma abordagem descritiva geral e uma análise comparativa dos macrodados estatísticos socioeconômicos das regiões Norte e Sul de Minas Gerais, na busca de uma possível relação com os dados de registro de violência doméstica contra a mulher nessas mesmas regiões. Os dados da violência doméstica foram obtidos a partir do Diagnóstico de Violência Doméstica e Familiar nas Regiões Integradas de Segurança Pública de Minas Gerais, referente ao ano 2018 publicado pelo governo do Estado. Os dados socioeconômicos foram obtidos no portal governamental Minas em Números.

Os números de registros de violência doméstica contra as mulheres divulgados no Diagnóstico são oriundos do denominado Armazém de Dados do Sistema Integrado de Defesa Social (SIDS)/Registro de Eventos de Defesa Social (REDS), que são os registros das ocorrências realizados pelos órgãos de segurança 
pública do Estado de Minas Gerais. Já os dados utilizados referentes aos indicadores socioeconômicos das regiões de planejamento são oriundos do portal Minas em Números, que tem por base as seguintes fontes: IBGE (2010), PNUD (2010), INEP Censo Escolar (2017), DATASUS (2017), FEAM-MG (2015), SEF-MG (2016), CAGED-MTE (2017) e Secretaria de Estado de Segurança Púbica de Minas Gerais - SESP/MG (2018).

As 19 RISPs estão delimitadas territorialmente dentro das regiões administrativas de Minas Gerais, mas não guardam uma uniformidade com estas e, por este motivo, ao buscar se comparar os dados referentes à segurança pública das RISPs e outros dados em âmbito regional depara-se com algumas limitações no que diz respeito ao recorte. No caso da região de planejamento Sul de Minas, as RISPs que se distribuem

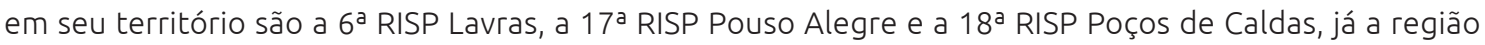
Norte de Minas tem em seu espaço territorial a presença da $11^{\text {a }}$ RISP Montes Claros.

Na região Sul, o número de municípios congregados pelas três RISPs citadas anteriormente ultrapassa o número dos municípios da região de planejamento, portanto, optou-se por retirar os números de registros de violência doméstica referentes ao município de Passos/MG, integrante da $18^{\text {a }}$ RISP Poços de Caldas, uma vez que em relação às regiões de planejamento, o município de Passos/MG pertence à região denominada Sudoeste de Minas. Por meio desse recorte, será possível uma maior aproximação da representação da realidade dos registros de ocorrências das RISPs em adaptação à abrangência regional da região Sul. Já a região Norte de Minas e a $11^{\text {a }}$ RISP Montes Claros guardam uma quase total uniformidade no número de municípios abrangidos.

No corpo do trabalho, será calculada e definida a taxa de crimes de violência doméstica contra as mulheres na proporção de Violência Doméstica por 100 mil habitantes, o que possibilitará a análise e a correlação com os outros indicadores de cada uma das regiões de planejamento objetos de investigação deste trabalho, e a posterior comparação entre as regiões.

\section{REFERENCIAL TEÓRICO}

Violência, conforme Zaluar (1999), pode ser entendida etimológica e conceitualmente e de forma aplicada como a prática de um ato de exercício de força para exercer a perturbação; assim como o crime, a violência também pode variar histórica e culturalmente. Conforme o autor, o termo,

vem do latim violentia que remete a vis (força, vigor, emprego de força física ou os recursos do corpo para exercer sua força vital). Essa força torna-se violência quando ultrapassa um limite ou perturba acordos tácitos e regras que ordenam relações, adquirindo carga negativa ou maléfica. É, portanto, a percepção do limite e da perturbação (e do sofrimento que provoca) que vai caracterizar o ato como violento, percepção essa que varia cultural e historicamente. (ZALUAR 1999, p. 8).

A violência doméstica contra a mulher é considerada, conforme Cerqueira et al. (2015, p. 9), como sendo a "mãe de todas as violências".

A Lei Maria da Penha tipifica violência doméstica contra a mulher como "qualquer ação ou omissão baseada no gênero que the cause morte, lesão, sofrimento físico, sexual ou psicológico e dano moral ou patrimonial" e define doméstico como sendo o "espaço de convívio permanente de pessoas, com ou sem vínculo familiar, inclusive as esporadicamente agregadas" (BRASIL, 2011, p. 16). 
Condições precárias de renda, acesso à saúde, à educação e ao emprego não apenas são deflagradores de atos violentos nas mais diversas dimensões, mas também contribuem para o agravamento desses mesmos problemas que originam, e em alguns estudos, como em De Alcântara et al. (2018) em um município do Nordeste, as condições precárias estão presentes no perfil das vítimas de violência doméstica. No estudo de Ribeiro e Sanches (2005) foi apurado que as mulheres vitimadas ganhavam em média $44 \%$ dos rendimentos médios em relação às mulheres não vitimadas, apontando para uma relação entre renda e violência doméstica.

O trabalho de Martins (2017) apontou uma correlação entre o menor nível de escolaridade e a vitimização doméstica das mulheres. A autora ainda encontrou na análise que:

na vitimização por violência doméstica a partir da cor, as mulheres pardas e pretas totalizam mais de $60 \%$ das vítimas, o que se assemelha às demais formas de crime, principalmente furtos e roubos. A predominância da violência sobre mulheres não brancas pode ser dada por uma maior vulnerabilidade destas, já que no Brasil as mulheres brancas são mais escolarizadas e possuem maior nível de renda. (MARTINS, 2017, p. 34).

Martins (2017) afirma que pelos resultados obtidos em seus estudos é possível constatar que as mulheres vitimadas possuem menor escolaridade e consequentemente auferem renda média mais baixa, fazendo a correlação entre baixo nível de escolaridade, desemprego e baixa renda, demostrando uma associação entre renda per capita baixa e a ocorrência de vitimização por violência doméstica.

Dias (2017) buscou problematizar a relação entre a exclusão social e a violência doméstica. A autora cita alguns fatores que estão presentes tanto nos grupos que sofrem com a exclusão social quanto na vida dos atores envolvidos em violência doméstica, citando: habitação deficitária, condições de saúde precárias, baixo nível de escolaridade, desemprego e precariedade do trabalho, baixos rendimentos, ausência de equipamentos coletivos, entre outros.

Para De Lucena et al. (2017), a violência doméstica é compreendida como sinônimo de violência contra a mulher, o que a determina como sendo um fenômeno de múltiplas determinações. Para Bandeira (2014), os atos de violência doméstica contra a mulher trazem como pano de fundo motivações como: hierarquia de poder; desejos de dominação e aniquilamento do outro; hegemonia do poder masculino, que permeia as relações entre homens e mulheres o que pode ser utilizada algumas vezes, conscientemente nas relações conjugais como mecanismos para subordinação da mulher ao parceiro.

O estudo de De Lucena et al. (2017) com 427 mulheres buscou por meio de uma análise regressiva a relação de dependência entre a qualidade de vida e a violência doméstica, e mostrou que quanto maior a avaliação da satisfação da qualidade de vida entre as mulheres, sua inserção em relações sociais e a sensação de segurança das mesmas menor será a probabilidade de ocorrência de violência doméstica contra a mulher, compreendendo-se como qualidade de vida o que é definido pela Organização Mundial de Saúde (OMS), conforme o WHOQOL GROUP (1998), enquanto como direito de cidadania o que se refere à soma das condições econômicas, ambientais, científico-culturais e políticas, coletivamente construídas e postas à disposição dos indivíduos para que estes possam realizar suas potencialidades. Segundo De Lucena et al. (2017), a qualidade de vida é um fator de proteção contra a violência doméstica.

Na outra vertente de estudos que não encontram relação de dependência direta entre violência doméstica, baixa escolaridade e renda está o trabalho de Moreira et al. (2016), que não identificou o aumento de 
renda funcionando como um fator estanque para a violência. Os autores, pesquisando a respeito do tema, descobriram que mulheres jovens que passaram a receber o benefício do Bolsa Família possuíam maior probabilidade de se tornarem vítimas de violência doméstica.

Ribeiro e Sánches (2005 apud MARTINS, 2017) apesar de identificarem o fator baixa renda como mais presente entre as mulheres vitimadas, chamam a atenção para um fato relevante em seu trabalho, ao ressaltarem que a violência doméstica ocorre em todos os níveis sociais, no entanto, os registros de violência doméstica se dão em maior número entre mulheres com maiores renda e escolaridade, uma vez que elas reúnem condições mais favoráveis para a denúncia por possuirem menor grau de dependência do agressor.

\section{APRESENTAÇÃO E DISCUSSÃO DOS DADOS}

Para entender a abrangência deste estudo da violência doméstica e a necessidade de compreendê-la como um fenômeno que pode ser tratado em caráter regional, apresenta-se aqui a $11^{\mathrm{a}}$ RISP Montes Claros, que congrega 77 dos 86 municípios da região Norte do Estado de Minas Gerais, enquanto que a $6^{a}$

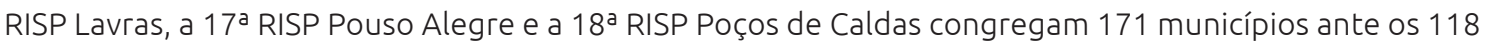
definidos como integrantes à região de planejamento Sul do Estado, ultrapassando, portanto, em 53 o número de municípios da região de planejamento. Destas três RISPs, toma-se aqui particularmente a $18^{a}$ RISP Poços de Caldas, cuja denominação da nomenclatura apresenta um município que está localizado na região Sul de Minas Gerais, mas é importante destacar que a 18 RISP abrange diversos municípios da região Sudoeste do Estado e, após análise, optou-se por excluir dos números finais da $18^{a}$ RISP os registros de violência doméstica do município de Passos/MG, que é maior município da região Sudoeste e que sozinho representa, segundo o IBGE (2010), quase 20\% da população e índices relacionados à economia da região Sudoeste.

No Quadro 1 é apresentado alguns indicadores socioeconômicos das regiões Norte e Sul do Estado de Minas Gerais.

\section{QUADRO 1}

\begin{tabular}{l|c|c|c|c|c|c|}
\hline \multicolumn{2}{l|}{ Indicadores regionais do Sul e do Norte de Minas Gerais } \\
\hline Região & População & Pobres & Analfabetismo & Saneamento & $\begin{array}{c}\text { PIB per } \\
\text { capita R\$ }\end{array}$ & $\begin{array}{c}\text { Taxa de } \\
\text { desocupação }\end{array}$ \\
\hline Norte & $1.577,300$ & $53,5 \%$ & $16 \%$ & $38,6 \%$ & 13.857 & $9,05 \%$ \\
\hline Sul & 2.031 .229 & $25,6 \%$ & $7,3 \%$ & $83,3 \%$ & 27.407 & $5,31 \%$ \\
\hline
\end{tabular}

Fonte: Elaborado pelo autor com base em informações obtidas no portal governamental Minas em Números (2019), disponivel em: <http://www.numeros.mg.gov.br>.

O Quadro 2, a seguir, apresenta os dados da violência doméstica nas Regiões Integradas de Segurança Pública que são abrangidas pelas regiões de planejamento Norte e Sul de Minas Gerais. 
QUADRO 2

\begin{tabular}{|c|c|}
\hline \multicolumn{2}{|c|}{$\begin{array}{l}\text { Quantitativo de registros de crimes de violência doméstica das regiões Sul e Norte de Minas Gerais, } \\
\text { no ano de } 2018\end{array}$} \\
\hline Região Integrada de Segurança Pública & Violência Doméstica 2018 \\
\hline $6^{\text {a RISP Lavras }}$ & 6.400 \\
\hline $17^{\text {a }}$ RISP Pouso Alegre & 5.704 \\
\hline 18a RISP Poços de Caldas & $7.908-881=7.027$ \\
\hline $11^{\mathrm{a}}$ RISP Montes Claros & 8.518 \\
\hline $\begin{array}{l}\text { Total de registros de violência doméstica no } \\
\text { Estado de Minas Gerais em } 2018\end{array}$ & 144.957 \\
\hline
\end{tabular}

Fonte: Diagnóstico de Violência Doméstica e Familiar nas Regiões Integradas de Segurança Pública de Minas Gerais (2019).

O Quadro 2 apresentou os números de registros de violência doméstica referente ao ano de 2018 nas regiões Sul e Norte, além da totalidade desses registros no Estado de Minas Gerais.

Dos 144.957 registros de violência doméstica ocorridos em todo o Estado de Minas Gerais no ano de 2018, os registros somados para essa mesma modalidade criminosa pelas RISPs que representam a região Sul foram 19.131, sendo 6.400 registos pela 6 ${ }^{a}$ RISP Lavras, 5.704 pela $17^{a}$ RISP Pouso Alegre e 7.027 pela $18^{a}$ RISP Poços de Caldas, com média de 6.377 crimes de violência doméstica por RISP integrante da região Sul.

Os dados obtidos por meio do portal governamental Minas em Números (2019), que utiliza os dados do IBGE (2010), apresentam a região Sul como tendo 2.031.229 habitantes, integrada por 118 municípios e um percentual de 81,63\% dessa população vivendo em área urbana.

Já na região Norte, a $11^{\text {a }}$ RISP Montes Claros registrou o número de 8.518 crimes de violência doméstica contra a mulher. Conforme o portal Minas em Números (2019), a região Norte possui 1.577 .300 habitantes, é integrada por 86 municípios sendo que 69,49\% da população vive em área urbana.

Considerando-se os números de registros absolutos de cada RISP em separado percebe-se que esses registros, tanto na RISP da região Norte quanto nas da região Sul, apresentam uma variação percentual de aproximadamente 33\% entre a RISP com maior número de registros, que é a $11^{\text {a }}$ RISP Montes Claros na região Norte, em relação à RISP que registrou o menor número de ocorrências de violência doméstica

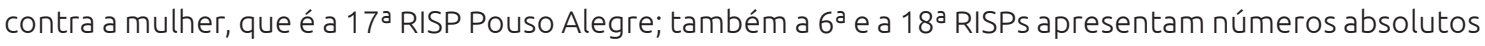
menores de registros do que a $11^{\text {a }}$ RISP. Os dados, quando apresentados em separado por RISP levam a um entendimento de que a região Sul teria o menor número de registros de violência doméstica se consideradas as RISPs descoladas do contexto regional das regiões de planejamento, no entanto, a análise individual por RISP cria distorções e barreiras para a análise regional do fenômeno da violência doméstica contra a mulher.

Um primeiro exercício de análise advém do número de crimes de violência doméstica dividido pela respectiva população das regiões estudadas, realizando-se o cálculo para obtenção do número de crimes de violência doméstica por 100 mil habitantes em cada região. Para se alcançar o índice de violência doméstica contra as mulheres por 100 mil habitantes utilizou-se a seguinte fórmula:

\section{FÓRMULA $=$ Registros de Violência Doméstica $=\Sigma$ ViolênciaDoméstica $=$ ViolênciaDoméstica/População x 100.000}


Após os cálculos, obtém-se o seguinte: a região Norte apresenta uma taxa de 540 crimes de violência doméstica para cada 100 mil habitantes, enquanto que a região Sul possui uma taxa de 981 crimes de violência doméstica por 100 mil habitantes, demonstrando, de acordo com o que é registrado pelos órgãos de segurança pública, que a taxa de crimes de violência doméstica nas regiões Sul de Minas é cerca de $81 \%$ maior do que na região Norte.

Tamanha diferença nos números de registros de crime de violência doméstica pode estar associada a fatores socioeconômicos, é o que se infere de Ribeiro e Sánchez (2005), quando afirmam que a renda e a escolaridade são dois indicadores socioeconômicos bastante relevantes quando se leva em conta os fatores de influência nas denúncias de violência doméstica às autoridades públicas e que, portanto, podem apresentar uma possível correlação direta com o número de registros. Segundo os autores, pessoas mais escolarizadas e com maior renda possuem melhor esclarecimento e menores custos associados para realizarem as denúncias; apontam ainda que em regiões onde os indicadores renda e escolaridade são maiores acontece um aumento no número de registros e denúncias aos órgãos de segurança diante da ocorrência de crimes de violência doméstica. Essa conclusão pode ser facilmente tomada a partir da comparação entre os Quadros 1 e 2 apresentados neste estudo.

A diferença muito relevante no número de registros, que aponta o índice da violência doméstica como sendo $81 \%$ maior na região Sul do Estado em relação à região Norte, pode ser associada também ao nível de analfabetismo das regiões; conforme o portal Minas em Números (2019), a região Sul possui a $6^{a}$ menor taxa de analfabetismo do Estado de Minas Gerais, já a região Norte, ao contrário, dentre as 17 regiões, é a $3^{a}$ com maior número de pessoas analfabetas no Estado. Esses dados, assim como o dado de desempregados, demonstram uma grande discrepância da região Sul em relação à região Norte, enquanto a região Sul apresenta uma média de desocupação de 4,77\% de sua população, a região Norte apresenta 9,05\% de desocupação de sua força de trabalho. Fatores assim, conforme Ribeiro e Sánchez (2005), são indicadores socioeconômicos que influenciam nas denúncias por parte das vítimas em relação ao crime de violência doméstica.

PIB per capita é o indicador que mostra a riqueza total de uma região dividida pelo número de moradores, tendo a região Sul $05^{\circ}$ maior PIB per capita do Estado, enquanto a região Norte aparece apenas na $14^{a}$ posição dentre as 17 regiões de Minas Gerais. Ao se observar esse indicador, os dados apresentados demonstram uma relação direta entre um PIB per capita alto e o alto número de registros de violência doméstica. Na região Sul de Minas Gerais o PIB per capita é cerca de 81 \% maior do que o da região Norte, demonstrando um percentual semelhante ao número dos registros de crime de violência doméstica na região Sul em relação à região Norte, que também é de $81 \%$.

Conforme Ribeiro e Sánchez (2005), as vítimas de violência doméstica que têm uma menor dependência do agressor possuem uma maior capacidade de denunciar o crime. O que coincide com os dados da violência doméstica registrados e apresentados pelas RISPs em relação aos indicadores socioeconômicos das regiões de planeamento investigadas.

O Sul de Minas apresenta a $3^{a}$ menor taxa de desemprego do Estado, a região Norte, por outro lado, apresenta a $2^{\mathrm{a}}$ maior taxa de desemprego, ocupando a $16^{\mathrm{a}}$ posição no ranking das 17 regiões. Esses dados de desemprego possuem uma aproximação com outro indicador que é a taxa de pessoas consideradas pobres, conforme a classificação adotada pelo governo no portal Minas em Números (2019), onde temse como pobre quem recebe menos que meio salário mínimo, sendo que os indicadores apresentam a região Sul de Minas Gerais na $6^{a}$ colocação dentre as menores taxas de pobreza no Estado, enquanto 
que a região Norte, de acordo com a classificação adotada pelo portal, é a $2^{\mathrm{a}}$ região que concentra o maior número de pessoas pobres do Estado, do que se infere, ao se comparar essas duas regiões com seus indicadores socioeconômicos e com os registros de violência doméstica, que existe uma relação de dependência direta entre as denúncias e os indicadores mais positivos.

\section{CONSIDERAÇÕES FINAIS}

A região Sul de Minas, como área administrativa para a gestão da segurança pública, é coberta espacialmente, não hermeticamente, pelas $6^{a}, 17^{\text {a e }} 18^{\mathrm{a}} \mathrm{RISPs}$; já a região Norte de Minas tem em seu espaço geográfico a $11^{a} \mathrm{RISP}$, que abrange quase todos os municípios da região.

Apesar das dificuldades para se elaborar o método e o recorte deste estudo, principalmente devido às diferenças entre as regiões adotadas pelo Estado para se definir os indicadores socioeconômicos e as regiões integradas para tratar e divulgar os números da segurança pública, foi possível por meio de uma análise comparativa entre as regiões e a correlação entre renda, escolaridade, pobreza, PIB e violência doméstica obter um resultado satisfatório ante o esforço realizado, quando se infere por meio do estudo que os registros de violência doméstica são realizados em maior número em regiões que possuem indicadores socioeconômicos mais favoráveis, mostrando uma relação de dependência direta entre rendas maiores e maior quantidade de registros de ocorrência de violência doméstica.

As regiões de planejamento foram delimitadas pelo IBGE (2017), considerando-se características de similaridades locais nos mais diversos setores, como econômicos, sociais e históricos. Chama a atenção, portanto, que seria de primeira ordem para o governo a aceitação da ocorrência da violência doméstica contra a mulher como um fenômeno de caráter regional, que acontece em localidades que guardam similaridades socioeconômicas, o que poderia facilitar a visão e as tomadas de ação por parte do Estado.

A violência doméstica contra a mulher é uma modalidade criminosa que foi negligenciada ao longo da história ao ser praticada em uma sociedade patriarcal e machista que julgava como um direito do homem a agressão contra a mulher. A partir de 2006, com a edição de legislação específica, a preocupação em prevenir e punir especificamente os crimes de violência doméstica passa a ser política de governo e meta dentro dos órgãos de segurança pública.

A literatura e os estudos empíricos ainda não pacificaram a dinâmica da influência de fatores econômicos e sociais sobre a prática da violência doméstica, mas no que diz respeito ao ato de se realizar a denúncia e o registro de tais crimes parece haver uma relação direta entre indicadores mais positivos e aumento nos números de registros, conforme recorte adotado neste trabalho.

O presente trabalho demonstrou que os números de registros de violência doméstica, comparando-se uma região com indicadores mais positivos e outra região com indicadores menos positivos, apresentam uma relação diretamente proporcional no que se refere aos registros oficiais de ocorrências dessa natureza, necessitando-se, no entanto, de um trabalho empírico para verificar a possibilidade de a realidade não oficial ser justamente o oposto.

A região do Estado de Minas Gerais que apresenta os maiores índices de analfabetismo, pobreza e menor renda é a região que apresenta uma taxa expressivamente menor de registros de crimes de violência 
doméstica por 100 mil habitantes, quando comparada com uma região que apresenta indicadores socioeconômicos mais favoráveis.

Vale ressaltar que o menor número de registros do crime de violência doméstica não representa necessariamente o baixo índice de ocorrência, podendo representar uma subnotificação dos fatos aos órgãos de segurança pública em razão das particularidades.

Por meio dos dados apresentados e discutidos, as contribuições deste estudo podem ser a de se constituir para além de uma base e um direcionamento para outros estudos da mesma natureza, colaborando igualmente para a chamada de atenção do governo na adoção de políticas públicas na abordagem e no tratamento da violência doméstica como fenômeno regional imbricado com outras variáveis, indicadores e particularidades, e não apenas pelo viés e pela divisão hermética da segurança pública, podendo-se a partir de estudos empíricos identificar e delinear melhor o problema.

Como sugestão para um trabalho futuro, propõe-se que os dados condensados e apresentados neste trabalho, que foi realizado a partir de pesquisa e grupamento dos indicadores e dos crimes de violência doméstica, possam ser tratados por meio de uma análise estatística regressiva multivariável ou ainda por meio de um estudo empírico que busque no campo a confrontação com os macrodados apresentados pelo governo.

\section{REFERÊNCIAS}

BANDEIRA, Lourdes Maria. Violência de gênero: a construção de um campo teórico e de investigação. Sociedade e Estado, v. 29, n. 2, p. 449-469, 2014.

BRASIL. Lei Maria da Penha e Legislação Correlata. Brasília: Senado Federal, Subsecretaria de Edições Técnicas, 2011. Disponível em: <http://www2.senado.leg.br/bdsf/bitstream/handle/ id/496319/000925795.pdf>. Acesso em: abr. 2019.

CERQUEIRA, Daniel et al. Avaliando a Efetividade da Lei Maria da Penha. Texto para discussão 2048. Brasília; Rio de Janeiro: Ipea, p. 1-36, mar. 2015. Disponível em: <http://www.ipea.gov.br/portal/images/ stories/PDFs/TDs/td_2048k.pdf>. Acesso em: 11 maio 2020.

DE ALCÂNTARA, Patrícia Pereira Tavares et al. Perfil da Mulher vítima de violência de gênero: um estudo documental. Revista E-Ciência, v. 6, n. 1, 2018.

DE LUCENA, Kerle Dayana Tavares et al. Associação entre a violência doméstica e a qualidade de vida das mulheres. Revista Latino-Americana de Enfermagem, v. 25, p. 1-8, 2017.

DIAS, Isabel. Exclusão social e violência doméstica: que relação?. Sociologia: Revista da Faculdade de Letras da Universidade do Porto, v. 8, 2017.

GERAIS, MINAS. Diagnóstico de violência doméstica e familiar nas Regiões Integradas de Segurança Pública de Minas Gerais. 2018. Disponível online no endereço: http://www.seguranca.mg.gov.br/ images/2019/Setembro/Diagnstico\%20violncia\%20domstica\%201\%20e\%202\%20Semestre\%20de\%20 \%202017\%20e\%20\%202018\%20e\%201\%20Semestre\%20de\%20219\%20-\%20MG\%20e\%20RISPs\%20 1\%201\%202.pdf. Acesso em: 23/02/2021

IBGE - Instituto Brasileiro de Geografia e Estatística. Censo demográfico 2010. IBGE, 2010. 
Divisão regional do Brasil em geográficas imediatas e regiões geográficas intermediárias: 2017. IBGE, Coordenação de Geografia. Rio de Janeiro: IBGE, 2017.

MACDONALD, Ziggy. Official crime statistics: their use and interpretation. The Economic Journal, v. 112, ก. 477, p. F85-F106, 2002.

MADALOZZO, Regina; FURTADO, Giovanna Maia. Um estudo sobre a vitimização para a cidade de São Paulo. Brazilian Journal of Political Economy, v. 31, n. 1, p. 160-180, 2011.

MARTINS, Jayne Cecília. Determinantes da violência doméstica contra a mulher no Brasil. 2017. Dissertação (Mestrado em Economia Aplicada) - Universidade Federal de Viçosa, Minas Gerais, Brasil. Disponivel em: <http://www.locus.ufv.br/handle/123456789/12860>. Acesso em: 23 de Fev de 2021

MINAS GERAIS. Minas em Números. A situação econômica e social de Minas Gerais. Disponível em: <http://www.seguranca.mg.gov.br/politica-de-privacidade/page/2500-estatisticas-criminais>. Acesso em: 20 fev. 2019.

MOREIRA, Gustavo Carvalho et al. Programa Bolsa Família e violência doméstica contra a mulher no Brasil. Estudos Econômicos, São Paulo, v. 46, n. 4, p. 973-1002, 2016.

RIBERO, Rocio; SÁNCHEZ, Fabio. Determinants, effects and costs of domestic violence. CEDE, v. $38,2005$. WHOQOL Group. The World Health Organization quality of life assessment (WHOQOL): Development and general psychometric properties. Social science \& medicine, v. 46, n. 12, p. 1569-1585, 1998.

ZALUAR, Alba. Um debate disperso: violência e crime no Brasil da redemocratização. São Paulo Perspec., v. 13, ก. 3, 1999.
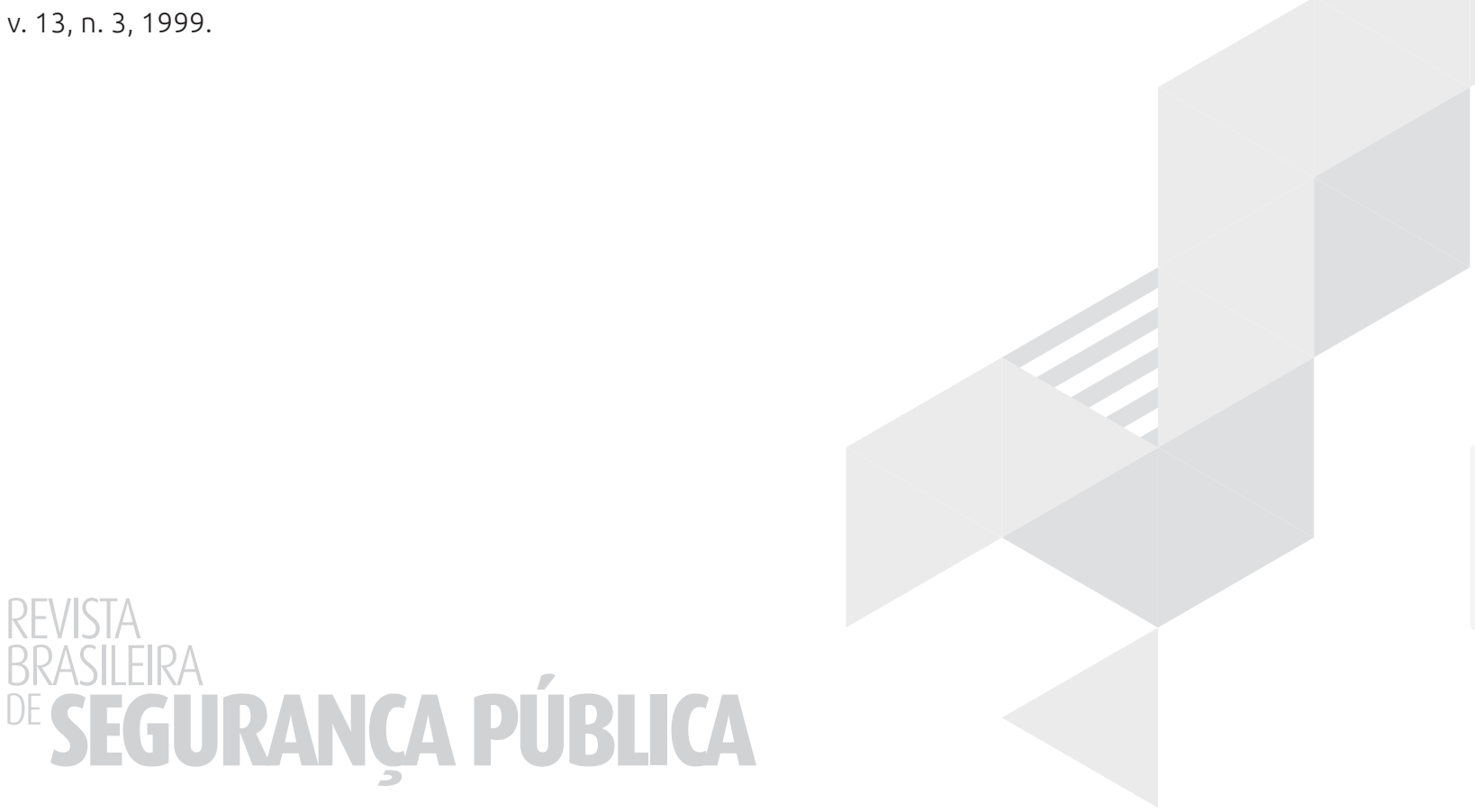